\title{
Reflection of nonlinear mountain waves by critical levels: behaviour of the reflection coefficient
}

Article

Accepted Version

Teixeira, M. A. C., Argain, J. L. and Xu, X. (2020) Reflection of nonlinear mountain waves by critical levels: behaviour of the reflection coefficient. Quarterly Journal of the Royal Meteorological Society, 146 (727). pp. 1009-1025. ISSN 1477870X doi: https://doi.org/10.1002/qj.3722 Available at https://centaur.reading.ac.uk/87875/

It is advisable to refer to the publisher's version if you intend to cite from the work. See Guidance on citing.

To link to this article DOI: http://dx.doi.org/10.1002/qj.3722

Publisher: Royal Meteorological Society

All outputs in CentAUR are protected by Intellectual Property Rights law, including copyright law. Copyright and IPR is retained by the creators or other copyright holders. Terms and conditions for use of this material are defined in the End User Agreement.

www.reading.ac.uk/centaur 
Central Archive at the University of Reading

Reading's research outputs online 


\title{
Reflection of nonlinear mountain waves by critical levels: behaviour of the reflection coefficient
}

\author{
M. A. C. Teixeira ${ }^{1} \quad$ J. L. Argaín ${ }^{2} \quad$ Xin $X^{3}$
}

${ }^{1}$ Department of Meteorology, University of Reading, Reading RG6 6BB, Berkshire, United Kingdom

${ }^{2}$ Department of Physics, University of Algarve, 8005-139 Faro, Algarve, Portugal

${ }^{3}$ School of Atmospheric Sciences, Nanjing University, Nanjing 210023, Jiangsu, China

\section{Correspondence}

Miguel A. C. Teixeira PhD, Department of Meteorology, University of Reading,

Reading RG6 6BB, Berkshire, United Kingdom

Email: m.a.teixeira@reading.ac.uk

Funding information
Critical levels, where the wind vanishes in the atmosphere, are of key importance for gravity wave drag parametrization. The reflectivity of these levels to mountain waves is investigated here using a combination of high-resolution numerical simulations and insights from linear theory. A methodology is developed for relating the reflection coefficient $R$ of 2D hydrostatic orographic gravity waves to the extrema of the associated drag as a function of an independent flow parameter. This method is then used to infer the variation of the reflection coefficient with flow nonlinearity. To isolate the effect of critical levels, a wind profile with negative shear is adopted, which is characterized by its Richardson number $R i$ and the dimensionless mountain height $N h_{0} / U_{0}$, based on the mountain height $h_{0}$, BruntVäisälä frequency $N$ and surface incoming wind speed $U_{0}$. Subject to the assumptions of linear theory, the drag is shown to be modified by wave refraction and reflection. The modulation of the drag by wave reflection is used to derive the reflection coefficient from the drag diagnosed from the numerical simulations. Despite considerable uncertainty, the critical level is found to have an $R$ that first increases with $N h_{0} / U_{0}$ for low values of this parameter, and for stronger nonlinearity saturates to a value of about 0.6 . The flow configuration in this saturated regime is characterized in the case of high-drag states by constructive wave interference, resembling downslope windstorms. Wave reflection by crit- 
ical levels enhances the flow nonlinearity and the associated drag amplification, more than doubling it for values of $N h_{0} / U_{0}$ as low as 0.12 . These results emphasize the need to represent this process in orographic gravity wave drag parametrizations, and suggest a possible way of doing it using a prescribed critical level reflection coefficient, derived using the present methodology.

\section{KEYWORDS}

Mountain waves, Gravity wave drag, Critical levels, Wave reflection, Nonlinear effects, Hydrostatic flow

\section{1 | INTRODUCTION}

Orographic gravity wave drag remains one of the key subgrid-scale physical processes that need to be parametrized in weather forecast and climate models (Teixeira, 2014). The importance of its representation, whose absence would decisively degrade the quality of forecasts and introduce spurious biases, was first shown compellingly by Palmer et al. (1986). In most existing orographic drag parametrizations, that force is represented in a very simplified way, typically ignoring wind profile effects on its net surface value (Lott and Miller, 1997), and often also neglecting nonlinear effects associated with high-amplitude mountain wave breaking, although a few exceptions regarding this last aspect do exist (Kim and Doyle, 2005). However, there are clear indications that both of these neglected effects may have a strong impact on the drag, under specific flow conditions. By its very nature, nonlinear drag amplification in highamplitude mountain waves gives a disproportionate contribution to the drag at the regional or global scales. Even in the linearized approximation, which provides a rough indication of the drag dependence on the flow parameters, the drag is proportional to the square of the orography elevation (Phillips, 1984), which skews its contribution towards high-amplitude waves. Miranda and James (1992) and Ólafsson and Bougeault (1996) explored the enhancement of the drag by flow nonlinearity, showing that for a value of the dimensionless mountain height of order one a wave breaking regime sets in, with amplification of the drag by a substantial factor relative to its reference value given by linear theory, even for an atmosphere with constant wind and static stability. The existence of shear considerably complicates this picture.

The importance of wave breaking for Clear-Air Turbulence generation in flows with directional shear has been investigated recently by Guarino et al. $(2016,2018)$ and Guarino and Teixeira (2017). Concurrently, it has been shown that the variation of the wind with height also leads to modifications to the net drag, either if this variation is gradual (Teixeira and Miranda, 2006, 2009; Teixeira and Yu, 2014), or more expressively if the variation is sudden, causing vertical reflection of the wave energy and some form of resonant wave interference (Teixeira et al., 2005, 2008). While these mechanisms are already present and quite important in linear flow, they become especially powerful in nonlinear flow, leading to amplification of the drag by factors of 2 or more, relative to its value in the absence of wave reflections (Miranda and Valente, 1997; Teixeira et al., 2005, 2008). Wave reflections and the attendant resonant drag enhancement may occur basically due to two physical mechanisms (understandable in a linear framework): either partial reflection, resulting from discontinuities in atmospheric parameters or their vertical derivatives (Wang and Lin, 1999; Teixeira et al., 2005, 2008), or total reflection at so-called turning-points, i.e., levels where the waves transition from being vertically-propagating to evanescent, originating trapped lee waves (Teixeira et al., 2013a; Yu and Teixeira, 
2015; Teixeira and Miranda, 2017).

Critical levels, where the wind speed vanishes and the wave equation becomes singular, are important for two reasons: they are heights where according to Eliassen-Palm's theorem the wave momentum flux may vary vertically even in steady and inviscid conditions (Eliassen and Palm, 1960), and they affect the net drag produced by mountain waves, as described next. Both of these effects are of key importance for gravity wave drag parametrization. Under the linear theory approximation, critical levels never lead to any wave reflection, but rather either transmit or absorb the waves, as shown originally by Booker and Bretherton (1967) and further elaborated on by Teixeira and Miranda (2009), Teixeira and Yu (2014) and Xu et al. (2012, 2013, 2017). However, as nonlinearity increases, this situation is thought to change, as suggested by various studies (Breeding, 1971; Clark and Peltier, 1984; Smith, 1985). It is especially difficult to disentangle the effects of wind profile variation and critical levels on drag behaviour, because typically the incoming wind profiles used to investigate flows with critical levels have variable shear, to ensure boundedness of the wind speed. For example, Miranda and Valente (1997), Wang and Lin (1999) and Teixeira et al. $(2005,2008)$ used piecewise-linear wind profiles to produce critical levels, whereas Scinocca and Peltier (1991), Lott (2007, 2016) and Damiens et al. (2018) adopted for the same purpose hyperbolic-tangent wind profiles. Qualitatively, both kinds of wind profiles have (smoothed or sudden) changes in their vertical shear, which affect the waves and hence the associated drag, either by refraction (in the former case) or more likely by reflection (in the latter case, but also in the former if the shear changes fast enough). Wave reflections at transitions in the vertical shear of the incoming wind have been invoked by Wang and Lin (1999) and Teixeira et al. $(2005,2008)$ to interpret the behaviour of the drag and wave structure. Whilst this approach was successful at low wave amplitudes, the behaviour of nonlinear flow pointed towards a more complicated role of critical levels, probably involving their transition from absorptive to reflective, confirming the initial conjectures of Breeding (1971) and Clark and Peltier (1984).

One of the few cases where the effect of a critical level may be isolated is that of a linear wind profile with constant negative shear. Although highly idealized, this profile has been adopted by Smith (1986), Grubišić and Smolarkiewicz (1997), Teixeira and Miranda (2006) and Teixeira et al. (2008) to understand gravity wave drag mechanisms. When this wind profile is adopted in numerical simulations, however, the wind velocity must typically be bounded, to avoid the risk of computational instability problems arising due to violation of the CFL condition. In such cases, it is usual to limit the wind magnitude at some height above the critical level, which of course implies a discontinuity or other variation of its vertical gradient (Teixeira et al., 2008). Nevertheless, this does not usually have a large impact on the wave characteristics below the critical level or the wave drag, since the critical level to a certain extent insulates the flow from what goes on above it due to its absorptive characteristics, as shown by Teixeira et al. (2008).

In the present study this simple linear wind profile will be used to investigate the reflective properties of critical levels in moderately nonlinear flow. Breeding (1971) was able to infer that the reflection coefficient of critical levels in nonlinear conditions increased with decreasing Richardson number $(R i)$, but given the limited number of cases considered, he was unable to establish a systematic dependence of the reflection coefficient on the nonlinearity parameter. On the other hand, the study of Teixeira et al. (2008) raised a number of intriguing questions about the behaviour of the drag as a function of both $R i$ and the dimensional mountain height. While for the wind profile with positive shear (without a critical level), the drag was seen to be amplified more relative to its value calculated from linear theory for low $R i$ than for high $R i$, for a wind profile with negative shear (and a critical level) the opposite was seen to occur. In particular, the amplification of the drag at the highest values of $R i$ considered did not approach the value predicted by weakly-nonlinear theory (Miles and Huppert, 1969) for zero shear (infinite Ri), the latter of which was nevertheless confirmed by numerical simulations for the same dimensionless mountain heights. In other words, for nonlinear flow the high- $R i$ limit, concerning drag behaviour, does not seem to approach the infinite- $R i$ limit. The purpose of the present contribution is to clarify this issue, in the simplest possible conditions that allow 
isolation of the relevant physical processes. With that aim, the focus will be on inviscid, non-rotating, hydrostatic flow, the latter assumption (conjugated with a wind profile with no curvature) automatically ruling out trapped lee waves. The chosen approach relies on a connection between the behaviour of the drag and the corresponding reflection coefficient, which is derived here and extrapolated from linear theory.

This paper is organized as follows: section 2 presents the theoretical framework used to interpret the results. Section 3 describes the numerical simulations used to represent mountain waves with varying degrees of nonlinearity. Section 4 presents the results and their interpretation, trying to clarify the dependence of the reflection coefficient of a critical level on nonlinearity for flows with relatively weak shear. Finally, section 5 summarizes the main conclusions of this study.

\section{2 | THEORY}

Inviscid, non-rotating, hydrostatic, steady waves generated by a 2D mountain ridge will be considered. For linearized flow with the Boussinesq approximation described in Fourier space, the Taylor-Goldstein equation reads:

$$
\hat{w}^{\prime \prime}+\left(\frac{N^{2}}{U^{2}}-\frac{U^{\prime \prime}}{U}\right) \hat{w}=0
$$

Here, $\hat{w}(k, z)$ is the Fourier transform of the vertical velocity perturbation ( $k$ is the horizontal wavenumber of the waves), $N$ and $U$ are the Brunt-Väisälä frequency and wind velocity of the mean incoming flow and the primes denote differentiation with respect to height, $z$.

It will be assumed that the wind near the surface, at least up to the critical level and possibly beyond, varies linearly with uniform negative shear such that

$$
U(z)=U_{0}-\alpha z
$$

where $U_{0}$ is the wind speed at the surface and $\alpha>0$ is a constant shear rate. For this wind profile, the term with the second derivative of the incoming wind in (1) vanishes, and the solution to that equation is of the form:

$$
\hat{w}(k, z)=\beta(k)\left(\frac{U(z)}{U_{0}}\right)^{\frac{1}{2}-i \mu s}+\gamma(k)\left(\frac{U(z)}{U_{0}}\right)^{\frac{1}{2}+i \mu s},
$$

where $s=\operatorname{sgn}(k), i=\sqrt{-1}, \mu=(R i-1 / 4)^{1 / 2}, R i=N^{2} / \alpha^{2}$ is the Richardson number of the incoming flow, and $\beta$ and $\gamma$ are coefficients to be determined that depend on the boundary conditions. Note that the first and second terms correspond to waves whose energy propagates upward and downward, respectively. Equation (3) may also be expressed in the form

$$
\hat{w}(k, z)=\beta(k)\left[\left(\frac{U(z)}{U_{0}}\right)^{\frac{1}{2}-i \mu s}+F(k)\left(\frac{U(z)}{U_{0}}\right)^{\frac{1}{2}+i \mu s}\right],
$$

where $F=\gamma / \beta$ may be related to a reflection coefficient, as will be seen.

The surface boundary condition, $\hat{w}(z=0)=i U_{0} k \hat{h}$, where $\hat{h}$ is the Fourier transform of the terrain elevation, 
implies that

$$
\beta=\frac{i U_{0} k \hat{h}}{1+F}
$$

On the other hand, the Fourier transform of the pressure perturbation associated with the waves is given by

$$
\hat{p}=\frac{i \rho_{0}}{k}\left(U^{\prime} \hat{w}-U \hat{w}^{\prime}\right),
$$

where $\rho_{0}$ is a reference (constant) density. If the solution to (1) in the form (4) is used, (6) may be written at the surface $(z=0)$ as follows:

$$
\hat{p}(z=0)=-\frac{\rho_{0} U_{0} \alpha \hat{h}}{1+F}\left[\frac{1}{2}(1+F)-i \mu s(1-F)\right] .
$$

Using Parseval's theorem, the orographic gravity wave drag on the 2D ridge (per unit length) may be expressed as (Teixeira, 2014)

$$
D=2 \pi i \int_{-\infty}^{+\infty} k \hat{p}^{*}(z=0) \hat{h} d k=-2 \pi i \int_{-\infty}^{+\infty} k \hat{p}(z=0) \hat{h}^{*} d k
$$

where the asterisk denotes complex conjugate. Inserting the definition of $\hat{p}(z=0)$ given by (7) into (8) yields

$$
D=2 \pi \rho_{0} U_{0} \alpha \int_{-\infty}^{+\infty} k|\hat{h}|^{2} \mu s \frac{1-F}{1+F} d k
$$

where use was made of the fact that the drag is a real quantity, and hence the first real term inside the square brackets in (7) gives no contribution.

In order to further simplify (9), two properties of $F(k)$ must be taken into account. Firstly, for hydrostatic flow $F$ is not actually a function of the magnitude of $k$, but only of its sign, i.e., $F=F(s)$. This is because (1) does not depend on $k$, and its solution (4) only depends on the magnitude of $k$ via the lower boundary condition, which is accounted for by (5). Since $F$ is the ratio of $\gamma$ and $\beta$, the dependence on $k$ of each coefficient cancels out. Secondly, $F$ satisfies $F^{*}(s)=F(-s)$, a property that will be used below. This results from (4)-(5) and from the fact that $w(x, z)$ is real, and so $\hat{w}^{*}(k, z)=\hat{w}(-k, z)$. Given this property, (9) may be rewritten as

$$
D=2 \pi \rho_{0} N U_{0} \frac{\mu}{R i^{1 / 2}} \int_{0}^{+\infty} k|\hat{h}|^{2}\left[\frac{1-F(s)}{1+F(s)}+\frac{1-F(-s)}{1+F(-s)}\right] d k .
$$

Since $F(s)$ is not a function of $k$, the expression in square brackets in (10) can be moved outside the integral. On the other hand, the drag for a constant wind equal to its surface value $U_{0}$ is

$$
D_{0}=4 \pi \rho_{0} N U_{0} \int_{0}^{+\infty} k|\hat{h}|^{2} d k
$$

which means that the drag (10) normalized by this value takes the form

$$
\frac{D}{D_{0}}=\frac{1}{2} \frac{\mu}{R i^{1 / 2}}\left[\frac{1-F(s)}{1+F(s)}+\frac{1-F^{*}(s)}{1+F^{*}(s)}\right]
$$


where the property of $F$ pointed out above has been used. Now, noting that the two terms in the square brackets in (12) are the complex conjugate of each other, that equation may be expressed further as

$$
\frac{D}{D_{0}}=\left(1-\frac{1}{4 R i}\right)^{1 / 2} \frac{1-|F|^{2}}{1+2 \operatorname{Re}(F)+|F|^{2}}
$$

where the definition of $\mu$ has also been used. If, as Teixeira et al. (2005) and Teixeira et al. (2008) did, a wave reflection coefficient is defined as $R=|F|$, then

$$
F=R e^{i \theta}=R(\cos \theta+i \sin \theta)
$$

where $\theta=\arctan [\operatorname{Im}(F) / \operatorname{Re}(F)]$, and (13) can be alternatively written

$$
\frac{D}{D_{0}}=\left(1-\frac{1}{4 R i}\right)^{1 / 2} \frac{1-R^{2}}{1+2 R \cos \theta+R^{2}}
$$

This equation makes it particularly transparent that the drag is affected by two distinct processes: wave refraction and wave reflection. Wave refraction is accounted for by the factor $[1-1 /(4 R i)]^{1 / 2}$ (this is the scaling of the drag for a linear wind profile that extends indefinitely (Smith, 1986, Teixeira and Miranda, 2004). The factor $\left(1-R^{2}\right) /\left(1+2 R \cos \theta+R^{2}\right)$, on the other hand, accounts for wave reflections. Note that it does not assume anything about the process that produces the reflection apart from the reflection coefficient $R$ and the phase of the reflection $\theta$. To isolate the effect of reflections, one may define

$$
D_{00}=D_{0}\left(1-\frac{1}{4 R i}\right)^{1 / 2}
$$

in terms of which (15) becomes simply

$$
\frac{D}{D_{00}}=\frac{1-R^{2}}{1+2 R \cos \theta+R^{2}}
$$

This perfectly isolates the effect of wave reflections. A formally similar expression applies in the case of a flow with no shear (and no critical level) in the region of interest, as is consistent with the explicit dependence of (15) on Ri.

If there is some process that causes the phase of the wave reflection to vary as a function of some independent flow parameter (for example, $R i$ ), such that the phase angle $\theta$ takes all possible values in the interval $[0, \pi]$ or spans an even wider interval, there will be some situations with $\theta=0 \Rightarrow \cos \theta=1$, and some situations with $\theta=\pi \Rightarrow \cos \theta=-1$. Then, according to (17), the drag takes, respectively, its minimum and its maximum values, given by

$$
\frac{D_{\min }}{D_{00}}=\frac{1-R}{1+R}, \quad \frac{D_{\max }}{D_{00}}=\frac{1+R}{1-R} .
$$

One consequence of this is that (18) may be inverted to obtain $R$ from the drag behaviour. In particular $R$ may be obtained either from $D_{\max } / D_{00}$ or $D_{\min } / D_{00}$ if $D_{00}$ is known, namely

$$
R=\frac{\frac{D_{\max }}{D_{00}}-1}{\frac{D_{\max }}{D_{00}}+1}=\frac{1-\frac{D_{\min }}{D_{00}}}{1+\frac{D_{\min }}{D_{00}}} .
$$


If, on the other hand, $D_{00}$ is not known beforehand, it can be determined from (18) as $D_{00}=\left(D_{\max } D_{\min }\right)^{1 / 2}$, and $R$ can also be determined directly from (18) in terms of $D_{\max } / D_{\min }$ as

$$
R=\frac{\left(\frac{D_{\max }}{D_{\min }}\right)^{1 / 2}-1}{\left(\frac{D_{\max }}{D_{\min }}\right)^{1 / 2}+1}
$$

Note that underlying all these manipulations is the assumption that $R$ does not change appreciably with the flow parameter that causes the drag modulation (mentioned above), particularly between its values for which $D_{\max }$ and $D_{\min }$ are attained. Once $R$ is known, $\theta$ may be determined in terms of $D / D_{00}$ as it varies with this flow parameter, via

$$
\cos \theta=\frac{1-R^{2}-\left(1+R^{2}\right) \frac{D}{D_{00}}}{2 R \frac{D}{D_{00}}} .
$$

Equations (20) and (21) allow a deduction of all parameters of the wave reflection simply from the behaviour of the drag, if this has discernible maxima and minima. Nothing is assumed about the nature of the process that causes the reflection itself. This provides a tool for evaluating the wave reflection coefficient and phase from the drag provided by generic numerical simulations of mountain waves where wave reflections are believed to occur. This tool will be used in the present paper with numerical simulations of flow with varying degrees of nonlinearity to try to infer the reflection properties of a critical level. A subtlety touched upon above is that, to find the extrema of $D / D_{00}$, its variation must be investigated as a function of some flow parameter (here $R i$ ), and it is possible that $R$ itself is a function of that same parameter (this is indeed the case in the simple example presented next). This precludes a perfectly accurate estimate of $R$ (as shown next). However, as long as $\theta$ varies faster with changes in $R i$ than $R$, the estimate of $R$ can still be quite accurate. Given that, in the parameter range that will be considered here, the extrema of $D / D_{00}$ are too sparse, no attempt will be made to estimate $\theta$, which would require a continuous description of the variation of both $D / D_{00}$ and $R$ with $R i$ in order to use (21).

\section{1 | Preliminary test of the method}

The feasibility and accuracy of the method outlined above is best tested using linear theory, in which analytical solutions of the flow exist and all the details that can be deduced from the method are known and may be checked. The wind profiles considered by Teixeira et al. (2008) are essentially similar to the one that will be used here: the wind speed either decreases linearly with height, reaching a critical level (as described by (2)), or it increases linearly instead, below a height where the shear changes discontinuously to zero. From the linear solutions, the first case, with negative shear (described by Figure 1(c) of Teixeira et al. (2008)), does not show any substantial wave reflection because of wave absorption by the critical level (that situation changes in nonlinear conditions, which are the focus here). But substantial wave reflection does occur (at the shear discontinuity) in the case with positive shear (described by Figure 1(a) of Teixeira et al. (2008)). The latter flow (similar to (2) near the surface, but with $\alpha<0$ ) will be considered in the tests presented next, since, from the above, it is irrelevant for the method what causes the wave reflection (either a shear discontinuity or a nonlinear critical level).

The reflection coefficient for this flow is given by (cf. Teixeira et al. (2008)):

$$
R=\left(\frac{1-\mu / R i^{1 / 2}}{1+\mu / R i^{1 / 2}}\right)^{1 / 2}
$$


Although Teixeira et al. (2008) did not calculate $\theta$, it may be shown that

$$
\theta=2 \mu \log \left|\frac{U_{1}}{U_{0}}\right|-\delta+\pi
$$

where $U_{1}$ is the wind velocity at the height where their wind profile becomes constant (see Figure 1(a) of Teixeira et al. (2008)), and $\delta=\arccos \left[1 /\left(2 R i^{1 / 2}\right)\right]$. If these two definitions are used in (17), an equation equivalent to the drag expression of Teixeira et al. (2008) for this case (their Eq. (17)) is recovered:

$$
\frac{D}{D_{00}}=\frac{\frac{\mu}{R i^{1 / 2}}}{1-\frac{1}{4 R i} \cos \left(2 \mu \log \left|\frac{U_{1}}{U_{0}}\right|\right)-\frac{\mu}{2 R i} \sin \left(2 \mu \log \left|\frac{U_{1}}{U_{0}}\right|\right)}
$$

Figure 1 shows $D / D_{00}$ (solid lines), $R$ (dashed lines) and $\cos \theta$ (dotted lines) obtained from (24), (22) and (23), respectively, as a function of $R i^{-1}$, for two different values of $\left|U_{1} / U_{0}\right|$ : 4 (Figure 1(a)) and 8 (Figure 1(b)). As can be seen (and was found previously by Teixeira et al. (2008)), $D / D_{00}$ oscillates with $R i^{-1}$, with the oscillations increasing in amplitude as $R i$ decreases. Clearly, this is due to the increase of $R$ as $R i$ decreases (see (22)), which reaches a value of 1 (perfect reflection) at $R i=1 / 4$. The oscillations of $D / D_{00}$ are closely connected with $\theta$. In particular, when $\theta=0 \Rightarrow \cos \theta=1$ the wave interference is destructive, leading to minima in $D / D_{00}$, whereas when $\theta=\pi \Rightarrow \cos \theta=-1$ the wave interference is constructive, leading to maxima in $D / D_{00}$ (as discussed above in generic terms). Perfect constructive wave interference and total reflection at $R i=1 / 4$ lead to an infinite value of $D / D_{00} . D / D_{00}$ and $\cos \theta$ are approximately in phase opposition (with maxima of $D / D_{00}$ corresponding to minima of $\cos \theta$ and vice-versa), but this correspondence is not perfect, because part of the modulation of $D / D_{00}$ is due to the variation of $R$. This is especially visible in the extrema on the right limit of the graphs, because that is where $R$ varies faster with $R i^{-1}$, and is an intrinsic limitation of the method adopted here.

Figure 2 shows $R$ (solid lines), $\left(D / D_{00}-1\right) /\left(D / D_{00}+1\right)$ (dashed lines) and $\left(1-D / D_{00}\right) /\left(1+D / D_{00}\right)$ (dotted lines) as a function of $R i^{-1}$ for the same values of $\left|U_{1} / U_{0}\right|$ as Figure 1 . According to (19), $\left(D / D_{00}-1\right) /\left(D / D_{00}+1\right)$ should coincide with $R$ at drag maxima and $\left(1-D / D_{00}\right) /\left(1+D / D_{00}\right)$ should coincide with $R$ at drag minima. The dots shown on the curves representing these quantities correspond to drag maxima and minima respectively. Clearly, the dots provide quite a good estimate (although a localized one in $R i$ parameter space) of the value of $R$. The quality of this estimate is slightly less good for the lowest values of $R i$ where $R$ varies faster with $R i^{-1}$, as pointed out above, but as long as the result that the phase varies faster than $R$ with $R i$ (which seems well fulfilled for high $R i$ ) can be extrapolated to nonlinear conditions, the method should work satisfactorily.

Table 1 shows a comparison between $R$ for the values of $R i$ for which $D / D_{00}$ attains extrema with the estimates obtained from (19), and the corresponding relative error. Only cases with $R i<64$ were included, since $R$ is otherwise quite small. It can be seen that the estimates are quite accurate, and only become less accurate for the lowest $R i$ where $R$ changes faster with $R i^{-1}$. Nevertheless, the error never rises above a few percent, which is good enough for the present purposes.

The method just developed and tested will be applied next to the output from numerical simulations, where, unlike in linear conditions, drag extrema exist for flows with negative shear. Note that, due to the fact that the dependence of $R$ on $R i$ is not known in that case, and $R$ is only estimated for discrete values of $R i$, it is not possible to infer the continuous variation of $\theta$ with $R i$, although it will be (reasonably) assumed that when $D / D_{00}$ is a maximum $\theta \approx \pi$ and when $D / D_{00}$ is a minimum $\theta \approx 0$. 


\section{3 | DESCRIPTION OF NUMERICAL SIMULATIONS}

Fully nonlinear 2D numerical simulations are carried out using the FLEX meso-to-microscale numerical model (Argaín, 2003), written in orthogonal curvilinear coordinates, which has been tested for a range of conditions, both inviscid (Teixeira et al., 2012, Teixeira et al., 2013a, Teixeira et al., 2013b) and including a full representation of boundary layer processes (Argaín et al., 2009, Argaín et al., 2017). The model is run in quasi-inviscid mode, i.e. using a Lilly subgridscale turbulence closure scheme, but a free-slip surface boundary condition. Since the incoming flow (described by (2) above) is dynamically stable, the turbulence closure is never activated except in regions of wave breaking and flow overturning. A grid of $200 \times 375$ points with spacings of $1760 \mathrm{~m}$ and $85.33 \mathrm{~m}$ (in the horizontal and vertical, respectively) is used in all simulations except those for the highest mountain, giving a domain width of $352 \mathrm{~km}$ (176 km to each side of the mountain) and a domain height of $32 \mathrm{~km}$. For the simulations with the highest mountain, the vertical grid comprises instead 444 points, giving a domain height of $38 \mathrm{~km}$. The shape of the lower boundary corresponds to a $2 \mathrm{D}$ bell-shaped mountain given by

$$
h(x)=\frac{h_{0}}{1+(x / a)^{2}},
$$

where $h_{0}$ is the maximum height and $a$ the half-width of the mountain. The mountain width is fixed at $a=16 \mathrm{~km}$ and the mountain height takes values between $h_{0}=10 \mathrm{~m}$ and $h_{0}=500 \mathrm{~m}$. The incoming flow is characterized by a constant Brunt-Väisälä frequency $N=0.01 \mathrm{~s}^{-1}$, a wind speed at the surface $U_{0}=10 \mathrm{~m} \mathrm{~s}^{-1}$, yielding a dimensionless mountain width $\mathrm{Na} / U_{0}=16$ (i.e. approximately hydrostatic flow), a dimensionless mountain height between $N h_{0} / U_{0}=0.01$ (strongly linear flow) and $N h_{0} / U_{0}=0.5$ (moderately nonlinear flow), and variable shear rates $\alpha$, corresponding to values of the Richardson number $R i=N^{2} / \alpha^{2}$ ranging from $R i=0.33$ to $R i=64$ (except for the $h_{0}=500 \mathrm{~m}$ mountain, in which case the upper limit is instead $R i=128$ ). The resolution is such that there are about 9 grid points per halfwidth of the mountain, and about 73 grid points per vertical wavelength of the wave based on the surface parameters: $\lambda_{z}=2 \pi U_{0} / N=6283 \mathrm{~m}$. Approximately 5.1 of these vertical wavelengths fit vertically into the computational domain, but the actual vertical wavelength is variable due to shear, approaching in particular zero at the critical level. This vertical resolution, while not very high, was nevertheless ensured to capture the dynamics of critical levels accurately. Tests at a resolution twice higher showed essentially similar results, with small (< $10 \%)$ departures, demonstrating the robustness of the adopted approach. Sponges are employed to damp spurious flow perturbations at the lateral boundaries of the domain, over a distance $2.5 \mathrm{a}$. Another sponge is applied at the top boundary of the domain to damp any wave reflections, having a thickness $1.5 \lambda_{z}$. The time step is $10 \mathrm{~s}$ and the simulation time has an average length of $160 a / U_{0}$ or 3 days, but has a fairly wide range of variation, depending on the flow conditions. Simulations are run until the drag stabilizes to a constant value for a period of at least several tens of $a / U_{0}$, which occurs in all runs. For computational stability reasons, the wind profile (2) needs to be limited above the critical level. This is done so that it never decreases below $U=-10 \mathrm{~m} \mathrm{~s}^{-1}$, i.e. $\left|U_{1} / U_{0}\right|=1$ in the notation of Teixeira et al. (2008). Tests made with $\left|U_{1} / U_{0}\right|=2$ showed little sensitivity to this parameter, something not unexpected because the critical level insulates the atmosphere above it from what goes on below, irrespective of whether it absorbs or reflects waves underneath.

\section{\begin{tabular}{l|l}
4 & RESULTS
\end{tabular}}

The quantity that will serve as the basis for all the results that follow is the steady-state drag produced in the numerical simulations described in the preceding section, particularly its maximum and minimum values as a function of $R i$. The 
fact that these drag extrema often occur at disparate values of $R i$ for a given value of $N h_{0} / U_{0}$, or at different values of $R i$ for different $N h_{0} / U_{0}$, carries with it the risk that it may be difficult to interpret systematically the variation of the drag (and of the reflection coefficient derived from it) as a function of $N h_{0} / U_{0}$, because of its (necessarily unknown) dependence on $R i$, but this is a risk that must be accepted for lack of a better alternative. Despite this risk, the results allow one to infer some interesting constraints and trends concerning the wave reflection process which substantially extend our current understanding of it.

Figure 3 shows the variation of $D$ given by FLEX normalized by $D_{00}$ defined by (16) with $R i$ for different values of $N h_{0} / U_{0}$ (see caption). When $N h_{0} / U_{0}$ is very low (e.g. 0.01 or 0.02 ), $D / D_{00}$ differs little from 1 , meaning that there is essentially no wave reflection. As $N h_{0} / U_{0}$ increases, maxima and minima of $D / D_{00}$ start to emerge and to depart progressively more from 1 , tending to occur at preferred values of $R i$. While for $N h_{0} / U_{0}$ between 0.01 and 0.08 , drag maxima occur for $R i=0.5$ or $R i=0.33$ (i.e. rather strong shear), for $N h_{0} / U_{0} \geq 0.1$ they occur for higher values of $R i$, ranging between $R i=4$ (for $N h_{0} / U=0.12$ ) and $R i=70$ (for $N h_{0} / U_{0}=0.5$ ). Drag minima tend to occur at a narrower range of $R i$, namely between $R i=11$ and $R i=14$ for $N h_{0} / U_{0} \leq 0.16$, and for somewhat higher values of $R i$ (up to 80) for $N h_{0} / U_{0}>0.16$. This distribution of the drag extrema determines the behaviour of the reflection coefficient, as will be seen next. A feature of the drag behaviour that stands out is that while drag minima are reasonably regular for all values of $N h_{0} / U_{0}$, drag maxima seem to undergo a regime transition from $N h_{0} / U_{0}=0.1$ to $N h_{0} / U_{0}=0.12$, with massive drag amplification $\left(D_{\max } / D_{00}>3.5\right)$ after this transition. This amplification is especially striking given that all considered values of $N h_{0} / U_{0}$ (up to 0.5 ) are quite moderate, or even small, and for a constant wind profile without a critical level, the drag is very little amplified relative to its value estimated from linear theory (as confirmed by Teixeira et al., 2008). On the other hand, especially for $N h_{0} / U_{0} \geq 0.16$, the drag displays a very sudden variation from its maximum (attained at a given value of $R i$ ) and much lower values below 1 in the vicinity of its minimum, for $R i$ only slightly higher. The $R i$ at which this jump takes place increases with $N h_{0} / U_{0}$, as can be seen in Figure 3 .

Figure 4 summarizes results for drag extrema and the values of the reflection coefficient derived from them. Figure 4(a) shows the drag maxima $D_{\max } / D_{00}$ and minima $D_{\min } / D_{00}$ as a function of $N h_{0} / U_{0}$ (irrespective of the value of $R i$ at which they occur), as well as the geometric mean of the two quantities, $\left(D_{\min } D_{\max }\right)^{1 / 2} / D_{00}$. According to section 2 , this should be taken as a reference drag in the absence of wave reflections, and in linear conditions it should take in particular the normalized value 1 . The dashed line in Figure 4 (a) corresponds to $D / D_{00}=1$ and the solid line denotes the nonlinear drag expression derived by Miles and Huppert (1969) for a flow without wind shear:

$$
\frac{D}{D_{00}}=1+\frac{7}{16}\left(\frac{N h_{0}}{U_{0}}\right)^{2}
$$

As can be seen in Figure 4(a), over the range of conditions simulated, the normalized drag maxima are always above 1 and the drag minima always below 1 , but whereas the drag minima have an almost monotonic behaviour, decreasing as $N h_{0} / U_{0}$ increases (except between 0.41 and 0.5 ), the drag maxima have a more irregular behaviour, with an initially smooth increase with $N h_{0} / U_{0}$, then a large jump between 0.1 and 0.12 (as mentioned above), an absolute maximum attained at $N h_{0} / U_{0}=0.16$, and for higher values of $N h_{0} / U_{0}$ stabilization at a value of $D_{\max } / D_{00} \approx 4$. This is reflected on the behaviour of $\left(D_{\min } D_{\max }\right)^{1 / 2} / D_{00}$, which is around 1 for $N h_{0} / U=0.01,0.02,0.04,0.08,0.1,0.32$ and 0.41 , but departs systematically towards higher values for $N h_{0} / U_{0}=0.12,0.16,0.23$ and 0.5 . This may be an indication either that the dependence of the reflection coefficient $R$ on $R i$ is too strong for drag extrema obtained at different values of $R i$ to be usable for inferring the dependence of $R$ on $N h_{0} / U_{0}$, or a sign that the process that is amplifying (or reducing) the drag in these flow configurations cannot be understood simply as a wave reflection, or possibly both reasons. Note that the value of drag amplification that would be expected from (26) purely due to nonlinear effects (i.e. in the absence of wave reflections) (solid line) is very modest, being only about $11 \%$ for $N h_{0} / U_{0}=0.5$. 
Figure 4(b) shows the reflection coefficient derived from the drag extrema displayed in Figure 4(a). The red symbols correspond to the reflection coefficient derived from drag maxima, $R_{\max }$, obtained from the first equation of (19), the blue symbols correspond to the reflection coefficient derived from drag minima, $R_{\min }$, obtained from the second equation of (19), and the black symbols correspond to the reflection coefficient derived from (20), $R_{i n t}$, equivalent to replacing $D_{00}$ by $\left(D_{\min } D_{\max }\right)^{1 / 2}$ in (19). If wave reflection was unquestionably the process leading to drag amplification or reduction in these numerical simulations, and if the drag maxima and minima were obtained for values of $R i$ for which $R$ did not differ, all three symbols should coincide. Their non-coincidence can in principle be ascribed to violation of either of these assumptions, although other explanations involving additional assumptions of the method described in section 2 cannot be ruled out. All the information contained in Figure 4 is summarized in numerical form in Table 2.

It can be seen in Figure 4(b) that $R_{\min }, R_{\max }$ and $R_{i n t}$ are fairly coincident up to $N h_{0} / U_{0}=0.08$, but start to diverge for $N h_{0} / U_{0}=0.1$, becoming highly discrepant for $N h_{0} / U_{0}=0.12,0.16$ and 0.23 , but coming again in closer agreement for $N h_{0} / U_{0}=0.32,0.41$, before finally diverging again for $N h_{0} / U_{0}=0.5$. Looking at the values of $R i$ at which the drag extrema occur (see Table 2), no obvious pattern is discernible relating the discrepancy between $R_{\max }, R_{\min }$ and $R_{i n t}$ and the values of $R i$ at which the corresponding drag maximum and minimum occur for each value of $N h_{0} / U_{0}$. Although, because of the disagreements just pointed out, substantial uncertainty exists in the diagnosed value of $R$, some broad trends are identifiable. Firstly, the reflection coefficient seems to increase roughly linearly for relatively low $N h_{0} / U_{0}$. Secondly $R$ seems to saturate to a constant value for $N h_{0} / U_{0}$ above a certain threshold. The first aspect is more apparent for $R_{\min }$, which perhaps can be attributed to the fact that the value of $R i$ at which $D_{\min } / D_{00}$ occurs does not vary so much as $N h_{0} / U_{0}$ increases, being limited to the interval between $R i=11$ and $R i=21$ (the equivalent range for $D_{\max } / D_{00}$ is between $R i=0.33$ and $R i=43$ ). The portion of the $R_{\min }$ variation between $N h_{0} / U_{0}=0.01$ and $N h_{0} / U_{0}=0.23$ can be fairly well approximated by the relation $R_{\min }=2\left(N h_{0} / U_{0}\right)$. On the other hand, for relatively high $N h_{0} / U_{0}$ (and this can be most clearly seen from $R_{\max }$ due to the plateau reached by $D_{\max } / D_{00}$ in Figure 4(a), but also from $R_{\min }$ and $R_{i n t}$, at least for $N h_{0} / U_{0}=0.32,0.41$ ), the reflection coefficient appears to saturate at an asymptotic value of $R \approx 0.6$.

While the uncertainty implied by the range spanned by the different symbols might be ascribed to the rather disparate values of $R i$ for which drag extrema occur in the cases with $N h_{0} / U_{0}=0.12,0.16$ and to a lesser extent $N h_{0} / U_{0}=0.23$, this argument does not seem valid for the case with $N h_{0} / U_{0}=0.5$, where actually the drag maximum and minimum occur for apparently quite close values of $R i$. However, it might not be possible to carry over the argument from linear theory about the more gradual variation of $R$ than $\theta$ with $R i$ to this case, since the flow may have become too nonlinear to allow it. This speculation would need to be tested via a more thorough exploration of parameter space, which is beyond the scope of the present paper. Additionally, how 'close' two values of $R i$ are is an essentially subjective judgement, unless some rational way to quantify it is developed.

\subsection{Analysis of the flow structure}

An analysis of the flow structure is useful to attribute specific flow regimes to a given drag behaviour, and its associated wave reflection coefficient. Figure 5 illustrates snapshots of the steady-state flow configuration associated with every drag maximum and minimum value included in Figure 4(a). The panels are ordered by ascending value of $N h_{0} / U_{0}$ (i.e. increasing flow nonlinearity), and a pair of flow cross-sections is presented for each value of $N h_{0} / U_{0}$ : the first one for the drag maximum and the second one for the drag minimum. Displayed are the normalized streamwise velocity perturbation, $u /\left(N h_{0}\right)$ (colour contours) and the streamlines of the flow, obtained from isentropes since the flow is adiabatic (thin solid lines). The orography profile is shown in black and the horizontal dashed line represents the critical 
level in each flow. Apart from the fact that drag maxima occur for much lower critical levels than drag minima, the flow behaviour for $N h_{0} / U_{0}=0.01,0.02,0.04$ and 0.08 (Figure 5(a)-(h)) is unremarkable. Maximum values of the normalized streamwise velocity perturbation are uniformly $\approx 1.4$ in magnitude (i.e. for positive and negative lobes of the field of this variable). However, higher values of $u /\left(N h_{0}\right)$ occur near the ground, particularly in the lee of the mountain, for the drag maxima than for the drag minima. Since the flow is inviscid and therefore governed by Bernoulli's equation, this is consistent with differences in the pressure perturbation that cause the drag in each case, which are necessarily higher for the drag maxima than for the minima. In Figure 5(a),(c),(e),(g), there is a signature of gravity waves propagating in the atmosphere above the critical level (where the incoming wind is reversed), which is consistent (at least qualitatively) with the prediction from linear theory that the critical level transmits $4 \%$ of the wave energy for $R i=0.5$ and $17 \%$ of the wave energy for $R i=0.33$, but their amplitude is relatively weak. However, one aspect that is not predictable from linear theory is the increase of $R$ from almost zero to about 0.19 as $N h_{0} / U_{0}$ increases from 0.01 to 0.08 .

For $N h_{0} / U_{0}=0.1$, apart from the fact that $u /\left(N h_{0}\right)$ is higher at the surface in the lee of the mountain for the drag maximum than for the drag minimum, and that the drag maximum now occurs for a much higher critical level, not much remarkable exists. Of course the mountain waves have a substantially higher amplitude than for lower $N h_{0} / U_{0}$, but since both drag extrema occur in this situation for quite high values of $R i$, essentially no leakage of wave energy occurs to the region above the critical level, as would be expected from linear theory. For $N h_{0} / U_{0} \geq 0.12$, however, the flow radically changes qualitatively. Now, the situation associated with drag maxima is systematically characterized by a very fast layer of flow attached to the ground, with a region of flow stagnation above (Figure 5(k),(m),(o),(q),(s),(u)). In these situations, typically the flow perturbation $u /\left(N h_{0}\right)$ attains its maximum immediately above the ground, with magnitude $>2.6$, but in some cases reaching values as high as 3.2 or even 3.6 (see the results for $N h_{0} / U_{0}=0.16,0.23$ ). This kind of flow configuration, where a typical upstream-tilted structure of the gravity waves is no longer visible, at least in the regions downstream of the mountain, is strongly reminiscent of the flows associated with 'downslope windstorms' in the studies of Clark and Peltier (1984), Smith (1985), or Bacmeister and Pierrehumbert (1988), having an apparently 'hydraulic' character. The downstream extent of the layer of fluid attached to the ground (with high wind speed), as well as the flow stagnation region overlying it, increases with $N h_{0} / U_{0}$. The time it takes to attain a steady state of the drag in these simulations is roughly proportional to the downstream extent of these layers, which take several days to stabilize. Whether it makes sense to apply a concept of wave reflection to such flows is open to question, but it is certainly true that the value of $R$ is relatively well constrained for $N h_{0} / U_{0}=0.32,0.41$ (where such flow configuration does occur), and the critical level is in all of these cases (for which $R i$ is quite large) unable to transmit any wave energy to the region of the atmosphere above it, apparently because it completely either absorbs or reflects the waves in the region of the atmosphere below it. The behaviour of the flow for the drag minima for this range of $N h_{0} / U_{0}$ is not as spectacular, with considerably lower-amplitude waves (albeit sometimes still breaking see Figure $5(\mathrm{r}),(\mathrm{t}),(\mathrm{v}))$, but very little variation of the maximum values attained by $u /\left(N h_{0}\right)$. In these cases, this variable keeps on attaining maxima of $\approx 1.2-1.4$, but perhaps the most salient feature, as $N h_{0} / U_{0}$ increases from 0.12 to 0.5 is the progressively more pronounced symmetry of the flow. This is seen especially regarding the maximum of $u /\left(N h_{0}\right)$ overlying the mountain, which initially is located downstream of the mountain but tends to migrate towards the peak as $N h_{0} / U_{0}$ increases. This is perhaps the most unmistakable signature of wave reflection seen in Figure 5 , since Teixeira et al. (2005) have shown it to be characteristic of reflected waves with destructive interference (see their Figures 3(c) and 6(c)). Obviously, the strong wind in the lee of the mountain is associated with the observed maximum drag values, whereas this almost symmetric wind maximum tends to produce the lowest drag values instead. 


\subsection{Wind profiles over the mountain}

To better understand the flow regime transition that occurs in Figure 5 between $N h_{0} / U_{0}=0.1$ and 0.12 , it is useful to examine how the wind profiles directly above the orography $(x=0)$ are affected by the wave perturbations. Figure 6 shows the total horizontal wind velocity $(U+u)$ normalized by its surface value $U_{0}$ as a function of height (red lines) at the drag maxima and minima for cases with $N h_{0} / U_{0}=0.02,0.1,0.12,0.23$, at the same times as used for evaluating the drag (i.e. end of the simulations). These values of $N h_{0} / U_{0}$ represent conditions that are nearly linear, immediately before and after the regime transition, and with a relatively large wave amplitude, respectively. In the latter case, the relatively low value $N h_{0} / U_{0}=0.23$ is selected because the flow structure does not change qualitatively for higher $N h_{0} / U_{0}$ (see Figure 5). The incoming wind profile is presented in Figure 6 for reference as the blue line. Note that in Figure 6 height is normalized by the critical level height $z_{c}$, which differs markedly between different cases. For example, in the case considered in Figure 6(a), which corresponds to the flow field in Figure 5(c), the critical level is much lower than in the case of Figure 6(b), which corresponds to the flow field in Figure 5(d).

The results of Figure 6 confirm more distinctly the aspect already noted in Figure 5 that critical levels in the background flow prevent almost completely the propagation of mountain waves above them, even when these are of relatively high amplitude. In all graphs, there is a clear correspondence between the maxima and minima in the profiles and those shown in the corresponding $u /\left(N h_{0}\right)$ fields in Figure 5. The case $N h_{0} / U_{0}=0.02$ (Figure 6(a),(b)) shows very little departure of the wind profile over the mountain from its incoming form, due to the small amplitude of the waves. For $N h_{0} / U_{0}=0.1$ (Figure 6(c),(d)), the wave amplitude is much larger. Although the total wind speed $U+u$ oscillates and gets close to zero below the critical level, particularly for the drag maximum (Figure 6(c)), it does not quite reach zero, so the critical level associated with the total flow coincides with that of the background flow, located at $z=z_{c}$. For $N h_{0} / U_{0}=0.12$ (Figure 6(e),(f)), the situation changes qualitatively. While for the drag minimum (Figure 6(f)) the critical level is still unchanged, at the drag maximum (Figure 6(e)) the critical level of the total flow $U+u$ is translated downward by about $0.2 z_{c}$ relative to that of the background flow. This so-called self-induced critical level is probably the reason why the flow becomes much more accelerated near the surface downstream of the mountain (see Figure 6(k)), producing much higher drag. For a still higher value of $N h_{0} / U_{0}=0.23$, the wind profile corresponding to the drag maximum has a self-induced critical level at $z \approx 0.6 z_{c}$ (Figure 6(g)), which presumably is responsible for producing a similar effect on the flow and on the drag, whereas the wind profile corresponding to the drag minimum has no self-induced critical level (Figure 6(g)), although its amplitude is almost sufficient to produce one.

The existence of self-induced critical levels and the modified values of $R i$ that characterize them locally likely change the height from which waves are reflected, and the behaviour of this reflection, as suggested by Peltier and Clark (1979), but this phenomenon is difficult to understand and study systematically. It is noteworthy that selfinduced critical levels are confined spatially in the horizontal direction to the vicinity of the mountain, whereas critical levels in the background flow exist everywhere at a fixed height. Additionally, the interpretation of the effects of self-induced critical levels is somewhat confusing, since those levels include in their definition the wave field they are supposed to affect. For these reasons, the characteristics of the background atmospheric profile should still be what ultimately drives these processes from upstream, justifying the approach adopted here. Finally, it is worth noting that the flow perturbations in the high-drag cases are larger downstream of the mountain than directly over it, but it is perhaps more meaningful to think about what happens over the mountain as triggering the behaviour observed further downstream, hence the focus on $x / a=0$ here. 


\subsection{Comparison with linear theory}

Flow fields similar to those presented in Figure 5 may be calculated using a linear model assuming the wind profile (2) and implementing solutions of the form (3), such as used, for example, by Teixeira et al. (2005) and Teixeira et al. (2008). This is easily done based on (3) by taking into account mass conservation in 2D

$$
i k \hat{u}+\hat{w}^{\prime}=0
$$

to obtain $\hat{u}$ and then calculating the inverse Fourier transform to obtain the streamwise velocity perturbation. Other variables may be related to $\hat{w}$ in Fourier space, and inversely Fourier transformed to obtain useful fields in physical space, such as the buoyancy perturbation, which allows the calculation of isentropes. This is done in Figure 7 for the same values of $N h_{0} / U_{0}$ as considered in Figure 6.

Figure 7 presents a comparison, of the flow fields calculated from the FLEX model and from linear theory below the critical level (which now corresponds to the top of the displayed domain) side by side. It can be seen that for $N h_{0} / U=0.02,0.1$ (first two rows), the qualitative agreement is quite good. The flow structure typical of vertically propagating mountain waves can be seen, with a field of $u /\left(N h_{0}\right)$ and streamlines that tilt upstream. An obvious difference is that linear theory displays a much higher amplification of $u /\left(N h_{0}\right)$ (and of the streamline perturbation) as the critical level is approached (where $u /\left(N h_{0}\right)$ actually diverges in the linear approximation) than the numerical simulations. This is partly due to the limited vertical resolution of FLEX, and is dictated by the inevitable computational dissipation that accompanies it. A more subtle but relevant difference is that the numerical results corresponding to the drag maxima (Figure $7(\mathrm{a}),(\mathrm{c}),(\mathrm{e}),(\mathrm{g}))$ have a magnitude of $u /\left(N h_{0}\right)$ near the ground larger than linear theory, an aspect that obviously is accounted for by the (relatively modest) wave reflection they show in Figure 4(b). A striking qualitative change of flow regime occurs for $N h_{0} / U_{0}=0.12$ (Figure 7(i)) (as noted in the description of Figures 5 and 6), which is due to the sudden amplification of $u /\left(N h_{0}\right)$ and change in the wave structure, with creation of a flow stagnation region in the lee of the mountain and an accelerated flow beneath it near the ground. None of this flow structure, or the magnitude of the associated flow perturbations, are captured by linear theory (Figure 7(j)), where, on the contrary the flow retains the same structure as for lower values of $N h_{0} / U_{0}$, albeit with an increased amplitude of the streamline displacements. For the corresponding drag minima, on the contrary (Figure $7(\mathrm{k}),(\mathrm{I}))$, differences between numerical results and linear theory are relatively slight. Moving on to a more nonlinear flow, Figure 7(m)-(p) shows similar comparisons for $N h_{0} / U_{0}=0.23$. In the numerical simulations for the drag maximum (Figure $7(\mathrm{~m})$, (q)), the downslope windstorm region extends further downstream, and its intensity becomes larger. For the drag minimum (Figure 7(o)), the flow perturbations remain modest, but their maxima migrate towards the mountain top. The linear model (Figure $7(n),(p)$ ) is clearly unable to reproduce either of these features, due to lack of a mechanism for wave reflections, although obviously the low-amplitude flow that occurs at drag minima is approximated more closely. A curious feature to notice in these results is that the lowest region of flow overturning from linear theory is more pronounced in the case corresponding to the drag maximum than in that corresponding to the drag minimum. For example, in Figure $7(\mathrm{n})$ the minimum of $u /\left(N h_{0}\right)$ is below -1.8 , whereas in Figure $7(\mathrm{p})$ it is only below -1.6 (the actual difference is even more significant, because the level where it occurs in the first case is closer to the critical level and thus with a lower background wind speed - than in the second). This suggests that the degree of stability of the flow as predicted by linear theory may influence the way in which the flow evolves to different fully nonlinear states, as suggested by Teixeira et al. (2005), even if the final nonlinear states resulting from this bifurcation may have very little in common with the corresponding linear states. 


\section{5 | CONCLUDING REMARKS}

The aim of the present contribution was to investigate the reflection properties of a mountain wave critical level for moderately nonlinear flow. This is a long-standing question, since, on the one hand, linear theory excludes wave reflection as one of the processes taking place at critical levels, only predicting wave absorption or transmission (depending on the Richardson number of the incoming flow). On the other hand, existing numerical studies of this process have not treated it systematically enough, and thus were largely inconclusive regarding the behaviour of the reflectivity of critical levels as a function of flow nonlinearity.

An especially puzzling finding is that while relatively weak flow nonlinearity causes a small departure of orographic gravity wave drag from its linear estimate in the absence of critical levels, the drag amplification and/or reduction tends to be much more pronounced when a critical level does exist, even when it has a very high Richardson number. In this respect the limit of sheared flow with a critical level at very high $R i$ does not seem to approach the infinite- $R i$ limit (without a critical level). Part of the explanation may be that, no matter how linear the flow at the surface is, as one approaches a critical level nonlinearity always becomes strong due to the vanishing wind speed that occurs there.

To isolate the effect of a critical level from that of any sudden or gradual variation of the wind profile, a wind profile with negative shear was considered here. This has the advantage of reducing the relevant parameters of the incoming flow to two: the $R i$ of the wind profile, and a nonlinearity parameter formed with the surface wind: $N h_{0} / U_{0}$. A method was developed to relate the reflection coefficient of a gravity wave associated with a generic process that causes wave reflections to the surface drag. This method allows deriving the reflection coefficient from the extrema of the drag as a function of $R i$ for numerical simulations of hydrostatic flow over a 2D ridge.

Underlying the application of the method to output from numerical simulations with $N h_{0} / U_{0} \leq 0.5$ is the idea that the reflection coefficient varies more slowly with $R i$ than the phase of the reflection causing the drag extrema. The method was first tested for a flow with forward shear near the surface and zero shear above a certain height, for which linear theory predicts drag modulation due to wave reflection at the shear discontinuity, and both the reflection coefficient and the wave phase may be calculated analytically. The results confirm the above hypothesis, at least for linear flow.

The method was then applied to nonlinear flow with a critical level, which is known to become reflective. It was implied that the assumptions that a wave reflection coefficient can be related to the gravity wave drag and that this reflection coefficient varies with $R i$ more slowly than the wave phase extend to these conditions. The results suggest that the reflection coefficient of the critical level increases roughly linearly for low $N h_{0} / U_{0} \leq 0.3$, and for higher $N h_{0} / U_{0}$ saturates at a value of $\approx 0.6$. However, some uncertainty remains regarding the value of $R$ obtained from its various definitions derived using linear theory. This may be either because in nonlinear conditions it becomes harder to disentangle the dependencies of $R$ on $N h_{0} / U_{0}$ and $R i$, or due to more complicated intrinsically nonlinear reasons, for which no interpretative framework currently exists. Clearly, these dependencies still need to be investigated more extensively.

While the flow structure (but not the reflection coefficient) can be predicted reasonably well by linear theory for $N h_{0} / U_{0} \leq 0.1$, for higher $N h_{0} / U_{0}$ the drag displays a very sudden transition from its maximum to its minimum as $R i$ increases, at a value of $R i$ that itself becomes larger with $N h_{0} / U_{0}$. Hence the drag extrema used to infer the reflection coefficient can only be detected at very high $R i$ (i.e. very high critical levels). This seems to question the partial results of Breeding (1971), which pointed towards an increase of the reflection coefficient for low $R i$.

The flow structure associated with the drag maxima is characterized by a strongly accelerated wind downstream of the mountain (resembling a downslope windstorm), attached to the surface for a downstream distance that increases with $N h_{0} / U_{0}$. In the establishment of this flow regime, self-induced critical levels appear to play a role, by modifying 
the heights at which wave reflection occurs relative to the background flow. On the other hand, the drag minima, which occur for only slightly higher values of $R i$, correspond to much weaker winds, with a maximum almost over the peak of the mountain. This behaviour suggests a bifurcation separating these two flow regimes, that is very sensitive to the value or $R i$, i.e., the height of the critical level (possibly triggered by subtle differences in the corresponding flows predicted by linear theory).

The method developed in this paper for relating drag extrema and the wave reflection coefficient is especially powerful in 2D hydrostatic flow, because the absence of wave dispersion allows these two quantities to be related in a closed analytical form. Either the directional dispersion that exists in flow over 3D mountains or the dispersion associated with non-hydrostatic effects makes the reflection coefficient depend, respectively, on the wavenumber direction or on its value (Teixeira et al., 2005; Teixeira et al., 2013a), which complicates its relation to the drag (an integrated quantity). However, a similar concept might still be applicable to these situations, with some modifications.

The relevance of wave reflections at critical levels for gravity wave drag parametrization is evident. Inasmuch as wave reflections can amplify the drag caused by flow over mountains by factors of 2-4, their overall impact cannot be neglected. One way to represent this effect would be by prescribing a drag amplification consistent with a reflection coefficient parametrized according to the results presented here. A difficulty to be overcome is, however, the high sensitivity of the drag amplification to the wave phase. The fact that critical levels at high altitudes can affect the drag so substantially is an aspect that requires special attention, as the wind profiles that cause such high-drag states may be virtually indistinguishable at low levels from those that cause no drag enhancement, or even drag reduction.

Apart from the other simplifying assumptions adopted in this study (inviscid approximation, 2D geometry, hydrostatic flow), a noteworthy limitation is that all results are obtained for steady flows, some of which take a substantial amount of time to stabilize. Whether these flows may be realized in Nature depends on the persistence of the synoptic circulation incident on the orography.

\section{references}

Argaín, J. L. (2003) Numerical Modelling of Atmospheric Flow: Orographic and Boundary Layer Effects. Ph.D. thesis, University of Algarve, Faro, Portugal.

Argaín, J. L., Miranda, P. M. A. and Teixeira, M. A. C. (2009) Estimation of the friction velocity in stably stratified boundary-layer flows over hills. Bound. Layer Meteor., 130, 15-28.

Argaín, J. L., Teixieira, M. A. C. and Miranda, P. M. A. (2017) Estimation of surface-layer scaling parameters in the unstable boundary layer: implications for orographic flow speed-up. Bound. Layer Meteor., 165, 145-160.

Bacmeister, J. T. and Pierrehumbert, R. T. (1988) On high-drag states of nonlinear stratified flow over an obstacle. J. Atmos. Sci., 45, 63-80.

Booker, J. R. and Bretherton, F. P. (1967) The critical layer for internal gravity waves in a shear flow. J. Fluid Mech., 27, 513-539.

Breeding, R. J. (1971) A non-linear investigation of critical levels for internal atmospheric gravity waves. J. Fluid Mech., 50, 545-563.

Clark, T. L. and Peltier, W. R. (1984) Critical level reflection and the resonant growth of nonlinear mountain waves. J. Atmos. Sci., 41, 3122-3134.

Damiens, F., Lott, F., Millet, C. and Plougonven, R. (2018) An adiabatic foehn mechanism. Quart. J. Roy. Meteor. Soc., 144, 1369-1381.

Eliassen, A. and Palm, E. (1960) On the transfer of energy in stationary mountain waves. Geofys. Publ., 22, 1-23. 
Grubišić, V. and Smolarkiewicz, P. K. (1997) The effect of critical levels on 3d orographic flows: linear regime. J. Atmos. Sci., 54, 1943-1960.

Guarino, M.-V. and Teixeira, M. A. C. (2017) Non-hydrostatic effects on mountain wave breaking in directional shear flows. Quart. J. Roy. Meteor. Soc., 143, 3291-3297.

Guarino, M.-V., Teixeira, M. A. C. and Ambaum, M. H. P. (2016) Turbulence generation by mountain wave breaking in flows with directional wind shear. Quart. J. Roy. Meteor. Soc., 142, 2715-2726.

Guarino, M.-V., Teixeira, M. A. C., Keller, T. L. and Sharman, R. D. (2018) Mountain wave turbulence in the presence of directional wind shear over the rocky mountains. J. Atmos. Sci., 75, 1285-1305.

Kim, Y.-J. and Doyle, J. D. (2005) Extension of an orographic-drag parametrization scheme to incorporate orographic anisotropy and flow blocking. Quart. J. Roy. Meteor. Soc., 131, 1893-1921.

Lott, F. (2007) The reflection of a stationary gravity wave by a viscous boundary layer. J. Atmos. Sci., 64, 3363-3371.

- (2016) A new theory for downslope windstorms and trapped mountain waves. J. Atmos. Sci., 73, 3585-3597.

Lott, F. and Miller, M. J. (1997) A new subgrid-scale orographic drag parametrization: its formulation and testing. Quart. J. Roy. Meteor. Soc., 123, 101-127.

Miles, J. W. and Huppert, H. E. (1969) Lee waves in a stratified flow. part 4. perturbation approximation. J. Fluid Mech., 35 , 497-525.

Miranda, P. M. A. and James, I. N. (1992) Non-linear three dimensional effects on the wave drag: splitting flow and breaking waves. Quart. J. Roy. Meteor. Soc., 118, 1057-1081.

Miranda, P. M. A. and Valente, M. A. (1997) Critical level resonance in three-dimensional flow past isolated mountains. J. Atmos. Sci., 54, 1574-1588.

Ólafsson, H. and Bougeault, P. (1996) Nonlinear flow past an elliptic mountain ridge. J. Atmos. Sci., 53, 2465-2489.

Palmer, T. N., Shutts, G. J. and Swinbank, R. (1986) Alleviation of a systematic westerly bias in general circulation and numerical weather prediction models through an orographic gravity wave drag parametrization. Quart. J. Roy. Meteor. Soc., 112, 1001-1039.

Peltier, W. R. and Clark, T. L. (1979) The evolution and stability of finite-amplitude mountain waves. part ii: Surface wave drag and severe downslope windstorms. J. Atmos. Sci., 36, 1498-1529.

Phillips, D. S. (1984) Analytical surface pressure and drag for linear hydrostatic flow over three-dimensional elliptical mountains. J. Atmos. Sci., 41, 1073-1084.

Scinocca, J. F. and Peltier, W. R. (1991) On the richardson number dependence of nonlinear critical-layer flow over localized topography. J. Atmos. Sci., 48, 1560-1572.

Smith, R. B. (1985) On severe downslope winds. J. Atmos. Sci., 42, 2597-2603.

- (1986) Further development of a theory of lee cyclogenesis. J. Atmos. Sci., 43, 1582-1602.

Teixeira, M. A. C. (2014) The physics of orographic gravity wave drag. Front. Phys. - Atmos. Sci., 2, 43.

Teixeira, M. A. C., Argaín, J. L. and Miranda, P. M. A. (2012) The importance of friction in mountain wave drag amplification by scorer parameter resonance. Quart. J. Roy. Meteor. Soc., 138, 1325-1337.

- (2013a) Drag produced by trapped lee waves and propagating mountain and propagating mountain waves in a two-layer atmosphere. Quart. J. Roy. Meteor. Soc., 139, 964-981. 
- (2013b) Orographic drag associated with lee waves trapped at an inversion. J. Atmos. Sci., 70, 2930-2947.

Teixeira, M. A. C. and Miranda, P. M. A. (2004) The effect of wind shear and curvature on the gravity wave drag produced by a ridge. J. Atmos. Sci., 61, 2638-2643.

- (2006) A linear model of gravity wave drag for hydrostatic sheared flow over elliptical mountains. Quart. J. Roy. Meteor. Soc., 132, 2439-2458.

- (2009) On the momentum fluxes associated with mountain waves in directionally sheared flows. J. Atmos. Sci., 66, 34193433.

- (2017) Drag associated with 3d trapped lee waves over an axisymmetric obstacle in two-layer atmospheres. Quart. J. Roy. Meteor. Soc., 143, 3244-3258.

Teixeira, M. A. C., Miranda, P. M. A. and Argaín, J. L. (2008) Mountain waves in two-layer sheared flows: critical level effects, wave reflection and drag enhancement. J. Atmos. Sci., 65, 1912-1926.

Teixeira, M. A. C., Miranda, P. M. A., Argaín, J. L. and Valente, M. A. (2005) Resonant gravity-wave drag enhancement in linear stratified flow over mountains. Quart. J. Roy. Meteor. Soc., 131, 1795-1814.

Teixeira, M. A. C. and Yu, C. L. (2014) The gravity wave momentum flux in hydrostatic flow with directional shear over elliptical mountains. Eur. J. Mech. B - Fluids, in press.

Wang, T.-A. and Lin, Y.-L. (1999) Wave ducting in a stratified shear flow over two-dimensional mountains. part i: general linear criteria. J. Atmos. Sci., 56, 412-436.

Xu, X., Shu, S. and Wang, Y. (2017) Another look on the structure of mountain waves: a spectral perspective. Atmos. Res., 191, 156-163.

Xu, X., Wang, Y. and Xue, M. (2012) Momentum flux and flux divergence of gravity waves in directional shear flows over three-dimensional mountains. J. Atmos. Sci., 69, 3733-3744.

Xu, X., Xue, M. and Wang, Y. (2013) Gravity wave momentum flux in directional shear flows over three-dimensional mountains: Linear and nonlinear numerical solutions as compared to linear analytical solutions. J. Geophys. Res., 118, 7670-7681.

Yu, C.-L. and Teixeira, M. A. C. (2015) Impact of non-hydrostatic effects and trapped lee waves on mountain-wave drag in directionally sheared flows. Quart. J. Roy. Meteor. Soc., 141, 1572-1585. 
TABLE 1 Examples of estimation of $R$ from $\left(D_{\max } / D_{00}-1\right) /\left(D_{\max } / D_{00}+1\right) \operatorname{or}\left(1-D_{\min } / D_{00}\right) /\left(1+D_{\min } / D_{00}\right)$ and the corresponding error, for $R i<64$. Values in the table correspond to the dots in Figure 2. (a) $\left|U_{1} / U_{0}\right|=4$, (b) $\left|U_{1} / U_{0}\right|=8$.

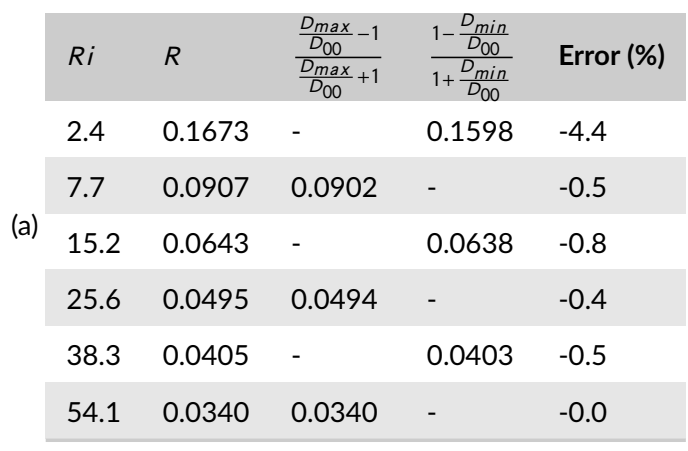

\begin{tabular}{lllll}
$R i$ & $R$ & $\frac{\frac{D_{\max }}{D_{00}}-1}{\frac{D_{\max }}{D_{00}}+1}$ & $\frac{1-\frac{D_{\min }}{D_{00}}}{1+\frac{D_{\min }}{D_{00}}}$ & Error (\%) \\
\hline 1.1 & 0.2565 & - & 0.2429 & -5.3 \\
3.5 & 0.1363 & 0.1355 & - & -0.6 \\
6.9 & 0.0962 & - & 0.0958 & -0.3 \\
11.4 & 0.0744 & 0.0741 & - & -0.4 \\
\hline
\end{tabular}

(b)

\begin{tabular}{lllll}
17.1 & 0.0607 & - & 0.0605 & -0.3 \\
23.9 & 0.0513 & 0.0510 & - & -0.6 \\
31.8 & 0.0444 & - & 0.0442 & -0.4 \\
41.0 & 0.0391 & 0.0390 & - & -0.3 \\
51.6 & 0.0348 & - & 0.0348 & -0.1 \\
62.8 & 0.0316 & 0.0316 & - & -0.1 \\
\hline
\end{tabular}


TABLE 2 Maximum and minimum drag for a range of $N h_{0} / U_{0}$, from the numerical simulations, values of $R i$ at which they occur, and values of $R$ derived from them.

\begin{tabular}{|c|c|c|c|c|c|c|c|}
\hline$\frac{N h_{0}}{U_{0}}$ & $R i$ & $\frac{D_{\max }}{D_{00}}$ & $\frac{D_{\min }}{D_{00}}$ & $\frac{\left(D_{\min } D_{\max }\right)^{1 / 2}}{D_{00}}$ & $R_{\max }=\frac{\frac{D_{\max }}{D_{00}}-1}{\frac{D_{\max }}{D_{00}}+1}$ & $R_{\text {min }}=\frac{1-\frac{D_{\min }}{D_{00}}}{1+\frac{D_{\min }}{D_{00}}}$ & $R_{i n t}=\frac{\left(\frac{D_{\max }}{D_{\min }}\right)^{1 / 2}-1}{\left(\frac{D_{\max }}{D_{\min }}\right)^{1 / 2}+1}$ \\
\hline 0.01 & 0.5 & 1.055 & - & 1.003 & 0.027 & - & 0.025 \\
\hline 0.01 & 11 & - & 0.953 & 1.003 & - & 0.024 & 0.025 \\
\hline 0.02 & 0.33 & 1.101 & - & 1.005 & 0.048 & - & 0.046 \\
\hline 0.02 & 11 & - & 0.917 & 1.005 & - & 0.043 & 0.046 \\
\hline 0.04 & 0.33 & 1.231 & - & 1.019 & 0.103 & - & 0.094 \\
\hline 0.04 & 14 & - & 0.844 & 1.019 & - & 0.085 & 0.094 \\
\hline 0.08 & 0.33 & 1.525 & - & 1.045 & 0.208 & - & 0.187 \\
\hline 0.08 & 14 & - & 0.716 & 1.045 & - & 0.165 & 0.187 \\
\hline 0.1 & 43 & 1.816 & - & 1.094 & 0.290 & - & 0.248 \\
\hline 0.1 & 14 & - & 0.659 & 1.094 & - & 0.206 & 0.248 \\
\hline 0.12 & 4 & 3.833 & - & 1.526 & 0.586 & - & 0.431 \\
\hline 0.12 & 14 & - & 0.607 & 1.526 & - & 0.244 & 0.431 \\
\hline 0.16 & 8 & 4.562 & - & 1.538 & 0.640 & - & 0.496 \\
\hline 0.16 & 14 & - & 0.519 & 1.538 & - & 0.317 & 0.496 \\
\hline 0.23 & 12 & 4.037 & - & 1.249 & 0.603 & - & 0.527 \\
\hline 0.23 & 18 & - & 0.387 & 1.249 & - & 0.442 & 0.527 \\
\hline 0.32 & 14 & 3.858 & - & 1.048 & 0.588 & - & 0.573 \\
\hline 0.32 & 21 & - & 0.285 & 1.048 & - & 0.557 & 0.573 \\
\hline 0.41 & 32 & 3.905 & - & 1.005 & 0.592 & - & 0.591 \\
\hline 0.41 & 37 & - & 0.259 & 1.005 & - & 0.589 & 0.591 \\
\hline 0.5 & 70 & 4.081 & - & 1.529 & 0.606 & - & 0.455 \\
\hline 0.5 & 80 & - & 0.573 & 1.529 & - & 0.272 & 0.455 \\
\hline
\end{tabular}



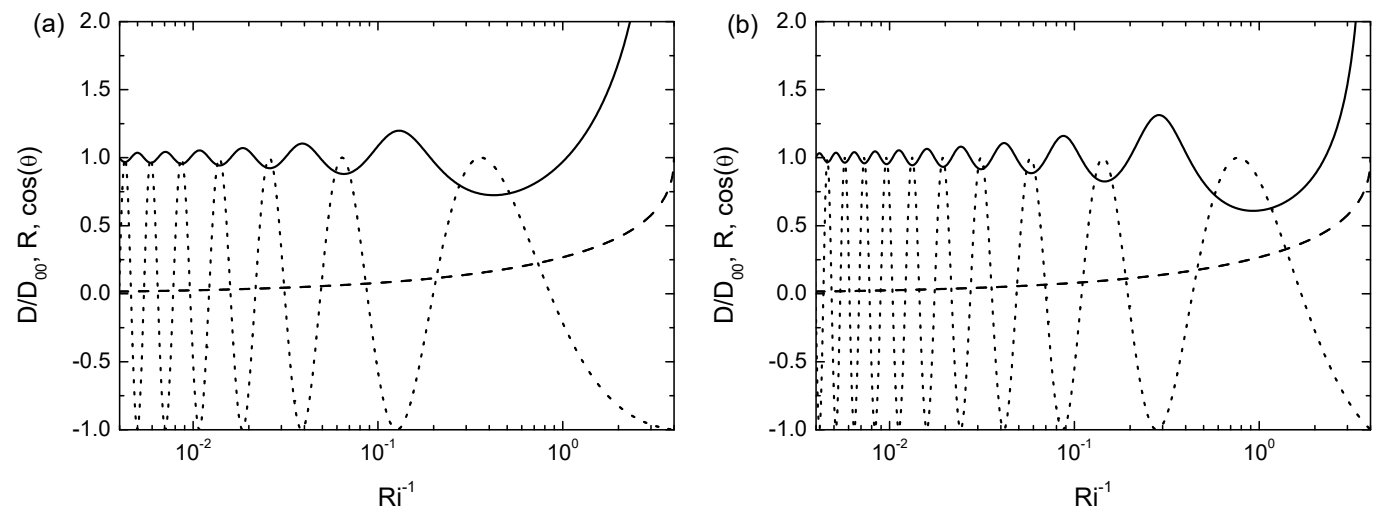

FIGURE 1 Normalized drag $D / D_{00}$ from (24) (solid line), reflection coefficient $R$ from (22) (dashed line) and $\cos \theta$ from (23) (dotted line) as a function of $R i^{-1}$, for the 2D flow with positive shear of Teixeira et al. (2008), from linear theory. (a) $\left|U_{1} / U_{0}\right|=4$, (b) $\left|U_{1} / U_{0}\right|=8$.
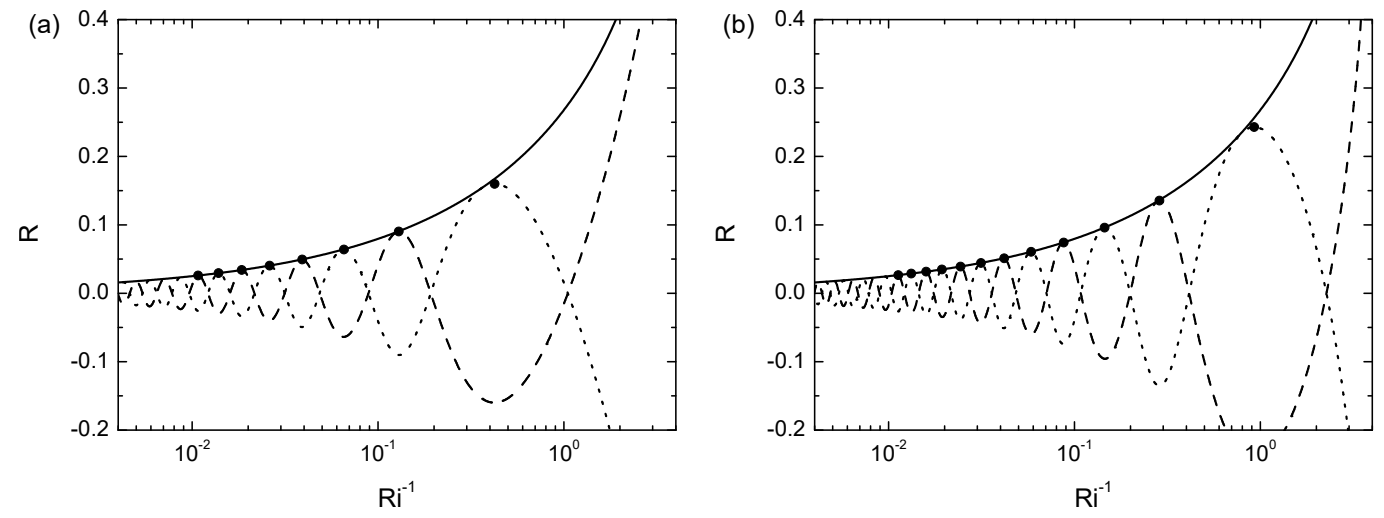

FIGURE 2 Reflection coefficient $R$ (solid line), $\left(D / D_{00}-1\right) /\left(D / D_{00}+1\right)$ (dashed line), $\left(1-D / D_{00}\right) /\left(1+D / D_{00}\right)$ (dotted line) (both quantities roughly coinciding at their maxima with $R$ ), and points corresponding to drag maxima and minima (dots), as a function of $R i^{-1}$, for the 2D flow with positive shear of Teixeira et al. (2008), from linear theory. (a) $\left|U_{1} / U_{0}\right|=4$, (b) $\left|U_{1} / U_{0}\right|=8$. 


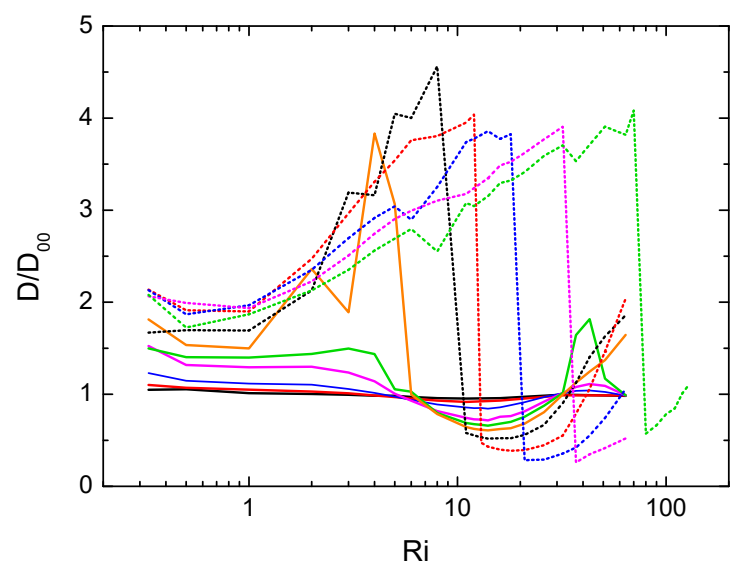

FIGURE 3 Normalized drag $D / D_{00}$ as a function of the Richardson number $R i$ for different values of the dimensionless mountain height $N h_{0} / U_{0}$. Solid black line: $N h_{0} / U_{0}=0.01$, solid red line: $N h_{0} / U_{0}=0.02$, solid blue line: $N h_{0} / U_{0}=0.04$, solid magenta line: $N h_{0} / U_{0}=0.08$, solid green line: $N h_{0} / U_{0}=0.1$, solid orange line: $N h_{0} / U_{0}=0.12$, dotted black line: $N h_{0} / U_{0}=0.16$, dotted red line: $N h_{0} / U_{0}=0.23$, dotted blue line: $N h_{0} / U_{0}=0.32$, dotted magenta line: $N h_{0} / U_{0}=0.41$, dotted green line: $N h_{0} / U_{0}=0.5$.
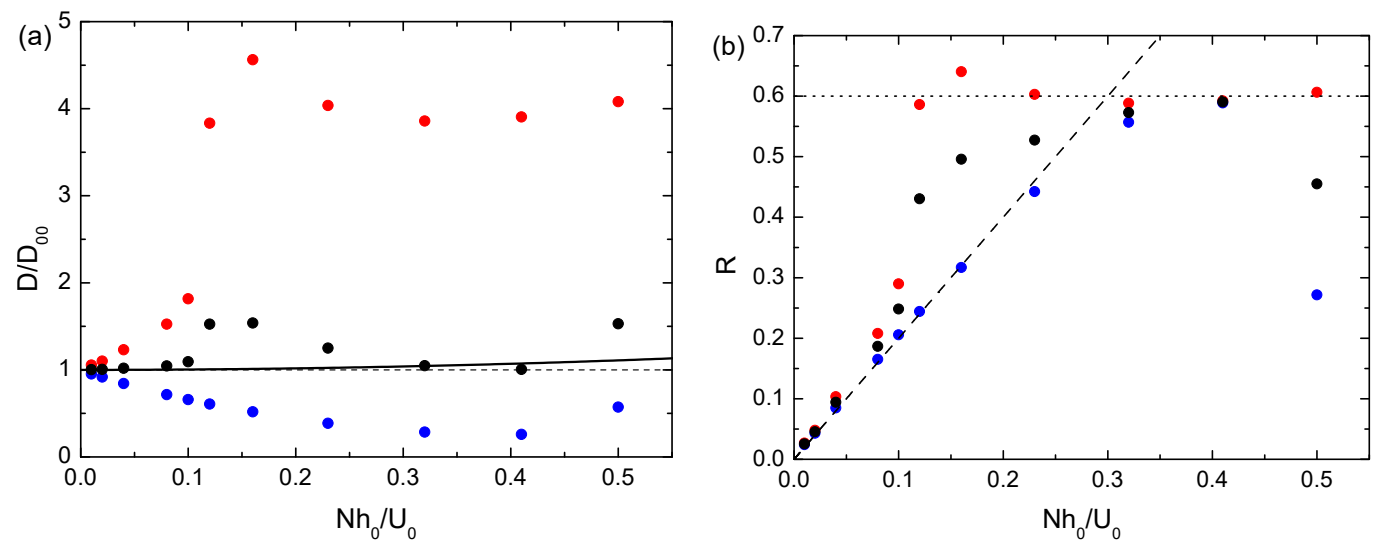

FIG URE 4 (a) Normalized drag maxima $D_{\max } / D_{00}$ (red circles) and minima $D_{\min } / D_{00}$ (blue circles) and their geometric average $\left(D_{\min } D_{\max }\right)^{1 / 2} / D_{00}$ (black circles) (in the range of $R i$ considered in Figure 3 ) as a function of the dimensionless mountain height $N h_{0} / U_{0}$. Dashed line: $D / D_{00}=1$, solid line: $D / D_{00}=1+(7 / 16)\left(N h_{0} / U_{0}\right)^{2}$. (b) Reflection coefficient as diagnosed from drag maxima, $R_{\max }$ (red circles), as diagnosed from drag minima, $R_{\min }$ (blue circles), given, respectively, by the first and second equations of (19), and as diagnosed from a combination of both, $R_{\text {int }}$ (black circles), given by (20). Dashed line: $R=2\left(N h_{0} / U_{0}\right)$, dotted line: $R=0.6$. 

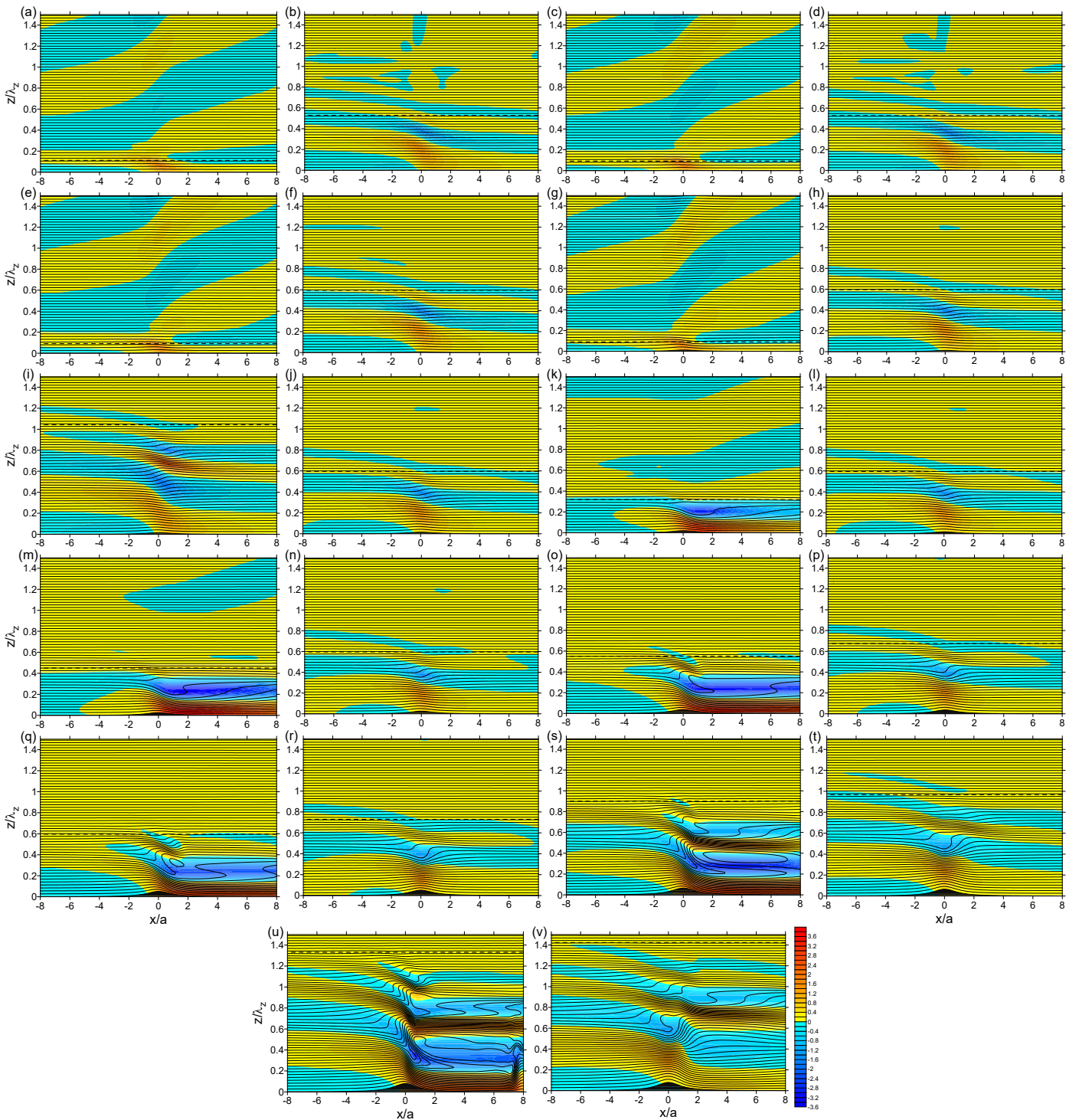

FIGURE 5 Cross sections of the flow for the situations associated with drag maxima ((a), (c), (e), (g), (i), (k), (m), (o), $(q),(s),(u))$ and drag minima ((b), (d), (f), (h), (j), (l), (n), (p), (r), (t), (v)) in Figure 3. Colour contours: normalized streamwise velocity perturbation $u /\left(N h_{0}\right)$ (see colour scale), thin black solid lines: lines of constant potential temperature (spacing $0.5 \mathrm{~K}$ ). Horizontal black dashed line: height of critical level $z_{c}$. Results for the following values of $\left(R i, N h_{0} / U_{0}\right)$ : (a) $(0.5,0.01)$, (b) $(11,0.01)$, (c) $(0.33,0.02)$, (d) $(11,0.02)$, (e) $(0.33,0.04)$, (f) $(14,0.04)$, (g) $(0.33,0.08)$, (h) $(14,0.08),(\mathrm{i})(43,0.1),(\mathrm{j})(14,0.1),(\mathrm{k})(4,0.12),(\mathrm{I})(14,0.12),(\mathrm{m})(8,0.16),(\mathrm{n})(14,0.16),(\mathrm{o})(12,0.23),(\mathrm{p})(18,0.23),(\mathrm{q})$ $(14,0.32),(r)(21,0.32),(s)(32,0.41),(t)(37,0.41),(u)(70,0.5),(v)(80,0.5)$. 

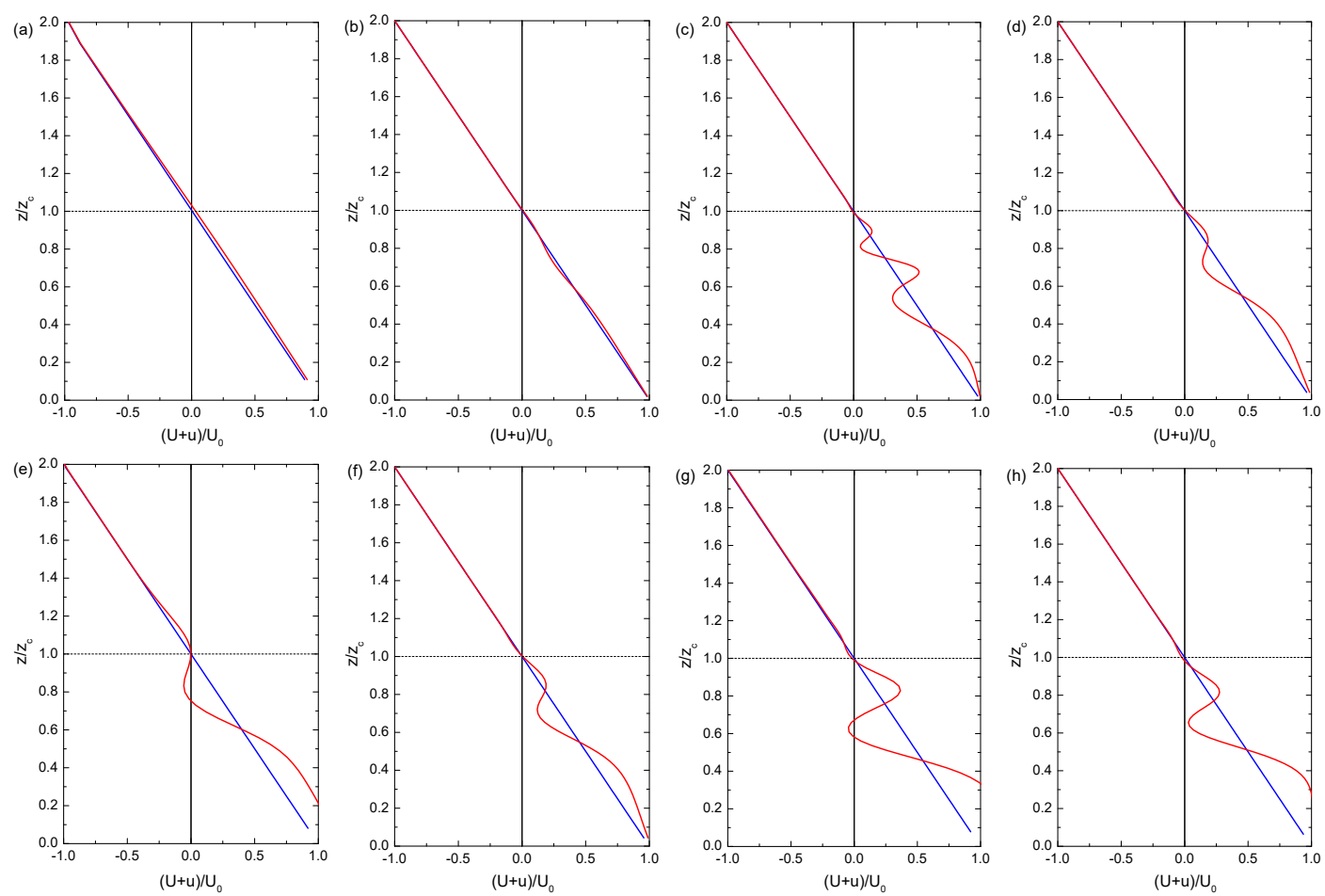

FIGURE 6 Total horizontal wind velocity $U+u$ normalized by the incoming wind velocity at the surface $U_{0}$ (red lines) as a function of height normalized by the critical level height $z_{c}$, at drag maxima ((a), (c), (e), (g)) and drag minima ((b), (d), (f), (h)). Results for the following values of $\left(R i, N h_{0} / U_{0}\right)$ : (a) $(0.33,0.02),(b)(11,0.02),(c)(43,0.1),(d)(14,0.1)$, (e) $(4,0.12)$, (f) $(14,0.12),(g)(12,0.23),(h)(18,0.23)$. Blue lines denote the incoming wind profile. 

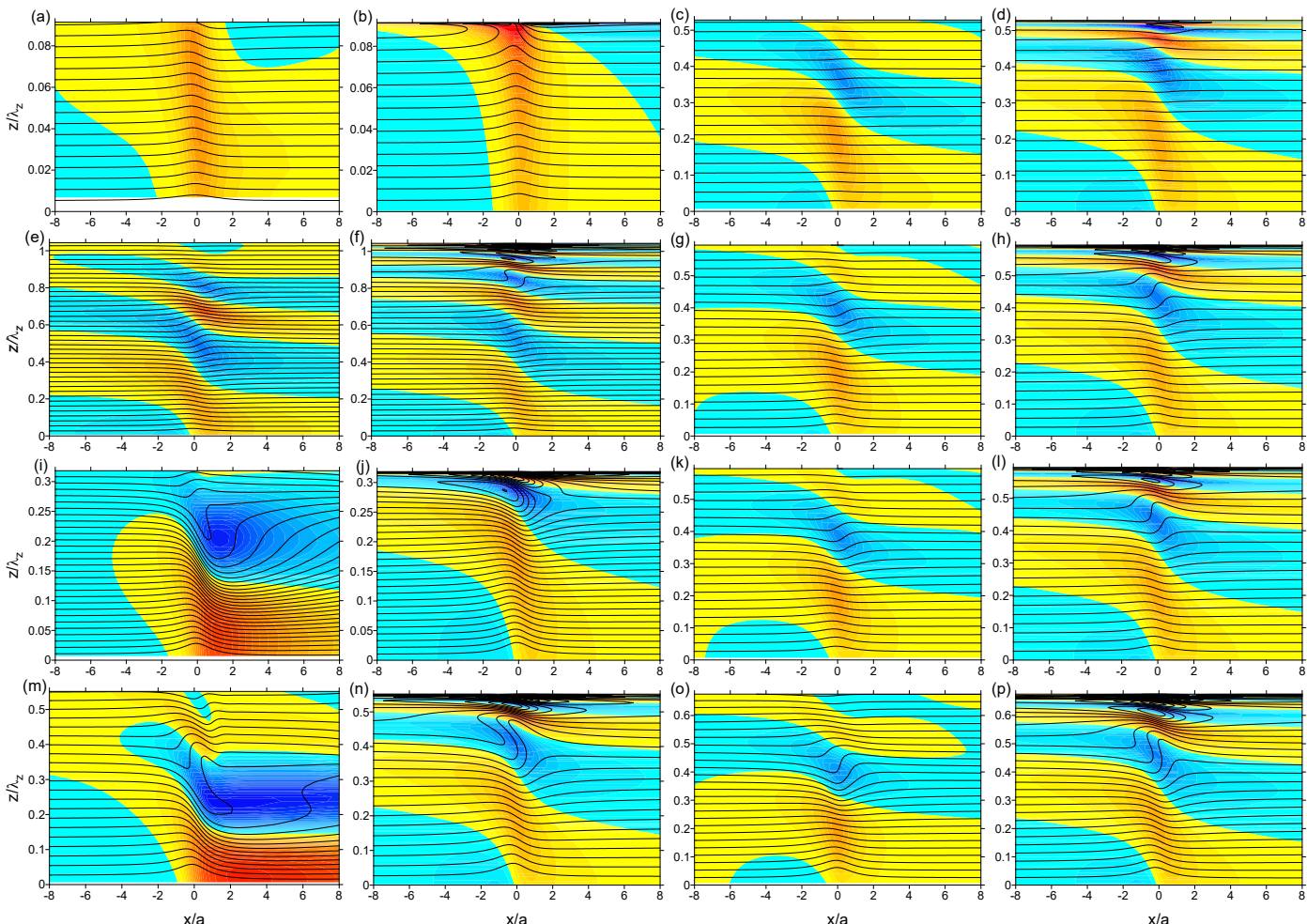

FIG URE 7 Comparison of flow cross-sections between numerical simulations ((a), (c), (e), (g), (i), (k), (m), (o)) and linear theory ((b), (d), (f), (h), (j), (l), (n), (p)), in the region of space below the critical level, for the drag maxima ((a), (b), (e), (f), (i), (j), (m), (n)) and minima ((c), (d), (g), (h), (k), (l), (o), (p)) selected in Figure 6. Colour contours: normalized streamwise velocity perturbation $u /\left(N h_{0}\right)$ (same colour scale as Figure 5 ), thin black solid lines: lines of constant potential temperature (variable spacing, but the same between matching numerical simulation and linear calculation). Results for the following values of $\left(R i, N h_{0} / U_{0}\right)$ : (a),(b) $(0.33,0.02),(\mathrm{c})$,(d) $(11,0.02),(\mathrm{e}),(\mathrm{f})(43,0.1),(\mathrm{g}),(\mathrm{h})(14,0.1)$, (i),(j) $(4,0.12),(\mathrm{k}),(\mathrm{l})(14,0.12),(\mathrm{m}),(\mathrm{n})(12,0.23),(\mathrm{o}),(\mathrm{p})(18,0.23)$. 